\title{
Discrete Global Sliding Mode Preestimate Congestion Control Algorithm for Internet
}

\author{
Yan Ming \\ College of Light Industry \\ Liaoning University \\ Shenyang, China \\ yanm0219@163.com \\ Liang Chao \\ College of Light Industry \\ Liaoning University \\ Shenyang, China \\ 1c000001@163.com
}

\author{
Liu Yandong \\ College of Light Industry \\ Liaoning University \\ Shenyang, China \\ liu198804@sina.com \\ Han Yezhong \\ College Of Physics \\ Liaoning University \\ Shenyang, China \\ hanyezhong@163.com
}

\begin{abstract}
In order to restrain the congestion phenomenon in Internet, a congestion control algorithm with state preestimate is proposed based on discrete global sliding mode control theory. Taking into account the uncertain and time-delay factors in actual Internet, a discrete global sliding surface with state preestimate is designed. Because the whole dynamics is global sliding mode, the robustness is obviously improved. Moreover, a new discrete reaching condition is presented. It can effectively restrain chattering. The proposed controller can obviously reduce the oscillation of the queue length in congestion router. The simulation results show that the proposed algorithm has better robustness. So it is better to adapt the effect of sudden data flows.
\end{abstract}

Keywords-Internet congestion control; discrete global sliding mode control; state preestimate; reaching condition

\section{INTRODUCTION}

It is well known that Internet is playing a more and more important role in society nowadays. Almost each national department cannot do without Internet. But Internet speed has been seriously reduced by congestion phenomenon. Only by introducing a congestion control mechanism, this problem can be solved.

In recent years, people had put forward a lot of congestion control algorithms [1-4]. By these algorithms, the congestion problem is restrained to a certain extent. However, because of the complexity and heterogeneity of Internet, uncertainty and time-delay factors widely exist in it. So it is urgent to find a congestion control algorithm with better robustness. The most major characteristics of sliding mode control is that the sliding mode dynamics possesses ideal robustness [5]. So it is very suitable for Internet congestion control. Sliding mode congestion control has become a hot research topic. Recently, there had been some algorithms [6-8]. But these references work based on continuous system. However, in fact, many devices in Internet are digital ones. Therefore, it is more practical to study on a discrete sliding mode congestion controller. Nowadays, some discrete sliding mode congestion control algorithms were also proposed [9, 10].
In [9], considering the dynamics of the window based TCP flow, a systematic control strategy based on discrete time sliding mode control was proposed. But there existed certain steady state oscillation in it. In [10], a discrete sliding surface was designed by linear matrix inequality (LMI), which compensated for the disadvantageous effect of uncertainties. But the input time-delay factor was not considered in it.

Because the whole dynamics is global sliding mode, global sliding mode control system is worthy to be mentioned. With global robustness, global sliding mode control system will greatly improve the effect of Internet congestion control.

In this paper, aiming at the congestion phenomenon in Internet, a discrete global sliding mode preestimate congestion control algorithm is proposed. The algorithm is presented based on a classical network congestion control model. Moreover, the uncertain and time-delay factors in system are also considered. So the model is closer to the actual Internet. A discrete global sliding surface with state preestimate is designed. The sliding surface can obviously improve the robustness of the system. In order to reduce the inherent chattering phenomenon in sliding mode control system, a new discrete reaching condition is also proposed. The controller that satisfied the reaching condition can effectively reduce the oscillation of the queue length in the congestion router. The simulation results show the effectiveness of the proposed algorithm.

\section{INTERNET CONGESTION CONTROL MODEL}

For transmitting data In Internet, about $95 \%$ of the data streams use transmission control protocol (TCP) as their transport layer protocol [11]. So it is rational to use a TCP network congestion control model to research Internet. In [1], a classical TCP network congestion control model was proposed as follows

$$
\delta \dot{W}(t)=-\frac{2 N}{R_{0}^{2} C} \delta W(t)-\frac{R_{0} C^{2}}{2 N^{2}} \delta p(t-R(t))
$$




$$
\delta \dot{q}(t)=\frac{N}{R_{0}} \delta W(t)-\frac{1}{R_{0}} \delta q(t)
$$

where $\delta W(t)=W(t)-W_{0}, W(t)$ is the current window size of TCP network, and $W_{0}$ is the expected value of $W(t) . \delta q(t)=q(t)-q_{0}, q(t)$ is current queue length of router, and $q_{0}$ is the expected value of $q(t) . N, C$ and $R_{0}$ are the nominal values of $N(t), C(t)$ and $R(t)$, respectively. $N(t)$ is the number of active TCP connections, $C(t)$ is the bandwidth of backbone link, $R(t)$ is round-trip time (RTT). $\delta p(t)=p(t)-p_{0}, 0 \leq p(t) \leq 1$ is the probability of packet marking/dropping, $p_{0}=2 N^{2} /\left(R_{0}^{2} C^{2}\right)$.

Let $x_{1}(t)=\delta q(t), x_{2}(t)=\dot{x}_{1}(t), u(t)=\delta p(t)$, So $u(t)$ satisfies $|u(t)| \leq 1, \tau=R(t)=(d-1) T+\tau_{1}$, where $0<\tau_{1}<T, d$ is an integer greater than $1, T$ is the sampling period of the digital system. Then (1) and (2) can be rewritten as

$$
\dot{\boldsymbol{x}}(t)=\boldsymbol{A} \boldsymbol{x}(t)+\boldsymbol{B} u(t-\tau)
$$

where $\boldsymbol{x}(t)=\left[\begin{array}{l}x_{1}(t) \\ x_{2}(t)\end{array}\right], \boldsymbol{A}=\left[\begin{array}{cc}0 & 1 \\ -\frac{2 N}{R_{0}^{3} C} & -\left(\frac{1}{R_{0}}+\frac{2 N}{R_{0}^{2} C}\right)\end{array}\right]$, $\boldsymbol{B}=\left[\begin{array}{c}0 \\ -\frac{C^{2}}{2 N}\end{array}\right],-p_{0} \leq u(t) \leq 1-p_{0}, 0 \leq p_{0} \leq 1$.

By [12], equation (3) can be discretized as follows

$$
\boldsymbol{x}(k+1)=\boldsymbol{G} \boldsymbol{x}(k)+\boldsymbol{H}_{1} u(k-d+1)+\boldsymbol{H}_{2} u(k-d)
$$

where $\boldsymbol{x}(k+1)=\left[\begin{array}{l}x_{1}(k+1) \\ x_{2}(k+1)\end{array}\right], \quad \boldsymbol{G}=\boldsymbol{e}^{\boldsymbol{A} T}, \quad T$ is the sampling period of the digital system, $\boldsymbol{H}_{1}=\int_{0}^{T-\tau_{1}} e^{A s} \boldsymbol{B} d s$, $\boldsymbol{H}_{2}=e^{\boldsymbol{A}\left(T-\tau_{1}\right)} \int_{0}^{\tau_{1}} \boldsymbol{B} d s$

However, actual Internet parameters often change, which are equivalent to the coefficient matrices $\boldsymbol{A}$ and $\boldsymbol{B}$ often change. So the coefficient matrices $\boldsymbol{G}, \boldsymbol{H}_{1}$ and $\boldsymbol{H}_{2}$ are often variable. So it is more rational to describe the actual Internet as follows

$$
\begin{aligned}
\boldsymbol{x}(k+1)= & (\boldsymbol{G}+\Delta \boldsymbol{G}(k)) \boldsymbol{x}(k)+\left(\boldsymbol{H}_{1}+\Delta \boldsymbol{H}_{1}(k)\right) u(k-d+1)+ \\
& \left(\boldsymbol{H}_{2}+\Delta \boldsymbol{H}_{2}(k)\right) u(k-d)
\end{aligned}
$$

where $\Delta \boldsymbol{G}(k)=\boldsymbol{G}(k)-\boldsymbol{G}, \Delta \boldsymbol{H}_{1}(k)=\boldsymbol{H}_{1}(k)-\boldsymbol{H}_{1}$ and $\Delta \boldsymbol{H}_{2}(k)=\boldsymbol{H}_{2}(k)-\boldsymbol{H}_{2}$ are the uncertain factors, $\boldsymbol{G}(k)$, $\boldsymbol{H}_{1}(k)$ and $\boldsymbol{H}_{2}(k)$ are the actual values of $\boldsymbol{G}, \boldsymbol{H}_{1}$ and $\boldsymbol{H}_{2}$, respectively.
Let

$\Delta \boldsymbol{\Phi}(k)=\Delta \boldsymbol{G}(k) \boldsymbol{x}(k)+\Delta \boldsymbol{H}_{1}(k) u(k-d+1)+\Delta \boldsymbol{H}_{2}(k) u(k-d)(6)$

So (5) can be rewritten as follows

$$
\boldsymbol{x}(k+1)=\boldsymbol{G} \boldsymbol{x}(k)+\boldsymbol{H}_{1} u(k-d+1)+\boldsymbol{H}_{2} u(k-d)+\Delta \boldsymbol{\Phi}(k)
$$

\section{DisCRETE GLOBAL SLIDING MODE PREESTIMATE CONGESTION CONTROL ALGORITHM}

\section{A. The Design of Discrete Global Sliding Surface}

A discrete global sliding surface is designed as follows

$$
S(k)=\boldsymbol{L} \boldsymbol{x}(k+d-1)+\boldsymbol{L H}_{2} u(k-1)-S_{0}
$$

where $S_{0}=\left(\boldsymbol{L} \boldsymbol{x}(d-1)+\boldsymbol{L H}_{2} u(-1)\right) \mathrm{e}^{-\lambda k}, \lambda$ is a small enough positive constant, $\boldsymbol{L}=\left[\begin{array}{ll}l_{1} & 1\end{array}\right], l_{1}>0$.

Because of $S(0)=0$, equation (8) is a discrete global sliding surface. By [13], we know that $S_{0}$ is a global sliding mode factor. So the dynamics on the discrete global sliding surface (8) is asymptotically stable.

Because the future state $\boldsymbol{x}(k+d-1)$ is in the discrete global sliding surface (8), it is essential to do state preestimate firstly.

The method of state preestimate is the same as [12], that is, we can obtain the value of the state $x(k)$ when time is $k$. In addition, we also record $u(k-1)$, $u(k-2), \cdots, u(k-d)$. So these future states $x(k+1)$, $x(k+2), \cdots, x(k+d-1)$ can be preestimated by (7).

\section{B. The Design of Discrete Reaching Condition}

This proposed discrete global sliding surface eliminates the reaching process. It means to eliminate the reaching process from the initial state to the discrete global sliding surface (8), and not to eliminate chattering phenomenon. Chattering phenomenon can be effectively restrained by designing a better reaching condition.

In [5], a discrete reaching condition was proposed as follows

$$
S(k+1)=(1-q T) S(k)-\varepsilon T \operatorname{sign} S(k)
$$

where $\varepsilon>0, q>0,0<q T<1, T$ is the sampling period.

The reaching time is finite and the chattering can be reduced by choosing appropriate $\varepsilon$ and $q$ [5].

When the system states are very close to their expected values, chattering phenomenon always exist because $\varepsilon T$ satisfies $\varepsilon T \neq 0$. Moreover, it is no way to obtain the optimal values of $\varepsilon$ and $q$ by [5].

The performance of a reaching condition directly affects the system performance. So it is necessary to design a better reaching condition. In order to overcome the above shortcomings of [9], we propose a new discrete reaching condition as follows

$$
S(k+1)=e^{-|S(k)|} S(k)-\left(1-e^{-|S(k)|}\right) \operatorname{sign} S(k)
$$


In (10), the function curves of $e^{-|S(k)|}$ and $1-e^{-|S(k)|}$ are as follows

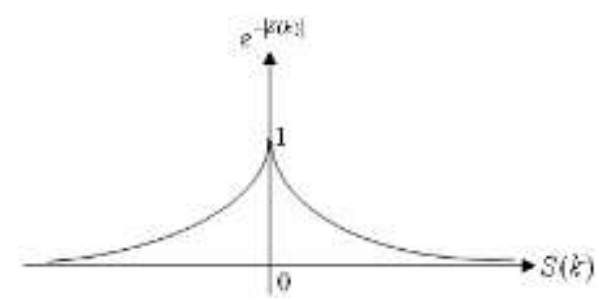

Figure 1. the function curve of $e^{-|s(k)|}$.

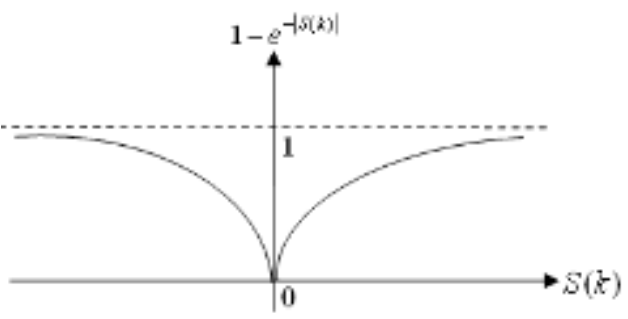

Figure 2. the function curve of $1-e^{-|S(k)|}$.

As is shown in Figure 1 and Figure 2, $e^{-|S(k)|}$ satisfies $0<e^{-|S(k)|} \leq 1 \quad, \quad$ and $1-e^{-|S(k)|} \quad$ satisfies $0 \leq 1-e^{-|S(k)|}<1$. When the system states are far away from the sliding surface, that is, when $|S(k)|$ is very large, $e^{-|S(k)|}$ is very small and $1-e^{-|S(k)|}$ is very close to 1 . The farther the system state is from the sliding surface, the bigger the rate of $|S(k+1)|$ becoming small, which can obviously increase the reaching rate. On the contrary, the closer the system state is from the sliding surface, the closer $e^{-|S(k)|} S(k)$ and $1-e^{-|S(k)|}$ are to 0 . So the reaching rate about the sliding surface is very low, that is, the inertia about the sliding surface is very small, so chattering phenomenon can be obviously restrained.

In a word, equation (10) can adaptively adjust reaching rate and effectively restrain chattering phenomenon. In addition, because $\varepsilon$ and $q$ do not exist in (10), it does not need to find the optimal values of $\varepsilon$ and $q$.

\section{The Design of Discrete Global Sliding Mode Conges- tion Controller}

In this subsection, we will design a discrete global sliding mode congestion controller, and we will make the proposed controller satisfy the reaching condition (10). So the controller can effectively restrain chattering phenomenon. That is, the proposed controller can effectively reduce the oscillation of the queue length in router.
Theorem 1: If the discrete global sliding mode congestion controller is as follows

$$
\begin{aligned}
u(k)= & \left(\boldsymbol{L H}_{\boldsymbol{1}}+\boldsymbol{L H}_{2}\right)^{-1}[-(\boldsymbol{L} \boldsymbol{G}+\boldsymbol{L}) \boldsymbol{x}(k+d-1)+ \\
& \boldsymbol{L} \boldsymbol{G} \boldsymbol{x}(k+d-2)-\left(\boldsymbol{L H}_{2}-\boldsymbol{L H}_{\boldsymbol{1}}\right) u(k-1)+ \\
& \boldsymbol{L H}_{2} u(k-2)+S_{1}+e^{-|S(k)|} S(k)- \\
& \left.\left(1-e^{-|S(k)|}\right) \operatorname{sign} S(k)\right]
\end{aligned}
$$

where $S_{1}=\left(\boldsymbol{L} \boldsymbol{x}(d-1)+\boldsymbol{L H}_{2} u(-1)\right) \mathrm{e}^{-\lambda(k+1)}$, Then the controller can satisfy the reaching condition (10), that is, it can effectively restrain chattering.

Proof: By (7), we have

$$
\begin{aligned}
& \boldsymbol{x}(k+d)= \boldsymbol{x}(k+1+d-1) \\
&= \boldsymbol{G} \boldsymbol{x}(k+d-1)+\boldsymbol{H}_{1} u(k)+ \\
& \boldsymbol{H}_{2} u(k-1)+\Delta \boldsymbol{\Phi}(k+d-1) \\
& \Delta \boldsymbol{\Phi}(k+d-1)=\boldsymbol{x}(k+d)-\boldsymbol{G} \boldsymbol{x}(k+d-1)- \\
& \boldsymbol{H}_{1} u(k)-\boldsymbol{H}_{2} u(k-1)
\end{aligned}
$$

In (13), $\Delta \boldsymbol{\Phi}(k+d-1)$ can not be obtained because $\boldsymbol{x}(k+d)$ is the future state. By (13), we have

$$
\begin{aligned}
\Delta \boldsymbol{\Phi}(k+d-2)= & \boldsymbol{x}(k+d-1)-\boldsymbol{G} \boldsymbol{x}(k+d-2)- \\
& \boldsymbol{H}_{1} u(k-1)-\boldsymbol{H}_{2} u(k-2)
\end{aligned}
$$

In (14), $\boldsymbol{x}(k+d-1)$ and $\boldsymbol{x}(k+d-2)$ can be obtained using state preestimate method in the previous subsection. So $\Delta \boldsymbol{\Phi}(k+d-2)$ can be obtained.

Let

$$
\Delta \boldsymbol{\Omega}=\Delta \boldsymbol{\Phi}(k+d-1)-\Delta \boldsymbol{\Phi}(k+d-2)
$$

Although $\Delta \boldsymbol{\Omega}$ is the uncertain item in system, its range is very small. This reason is that $\Delta \boldsymbol{\Omega}$ is the difference of two next sampling periods.

By (8), we have

$$
S(k+1)=\boldsymbol{L} \boldsymbol{x}(k+d)+\boldsymbol{L H}_{2} u(k)-S_{1}
$$

Substituting (12) into (16), (16) can be rewritten as follows

$$
\begin{aligned}
S(k+1)= & \boldsymbol{L} \boldsymbol{G} \boldsymbol{x}(k+d-1)+\boldsymbol{L H}_{1} u(k)+ \\
& \boldsymbol{L H}_{2} u(k-1)+\boldsymbol{L H}_{2} u(k)+ \\
& \boldsymbol{L} \Delta \boldsymbol{\Phi}(k+d-1)-S_{1}
\end{aligned}
$$

By (14) and (15), (17) can be rewritten as follows

$$
\begin{aligned}
S(k+1)= & (\boldsymbol{L} \boldsymbol{G}+\boldsymbol{L}) \boldsymbol{x}(k+d-1)-\boldsymbol{L} \boldsymbol{G} \boldsymbol{x}(k+d-2)+ \\
& \left(\boldsymbol{L H}_{1}+\boldsymbol{L H}_{2}\right) u(k)+\left(\boldsymbol{L H}_{2}-\boldsymbol{L H}_{1}\right) u(k-1)-(18) \\
& \boldsymbol{L H}_{2} u(k-2)-S_{1}+\boldsymbol{L} \Delta \boldsymbol{\Omega}
\end{aligned}
$$

Substituting (11) into (18), (18) can be rewritten as follows

$$
S(k+1)=e^{-|S(k)|} S(k)-\left(1-e^{-|S(k)|}\right) \operatorname{sign} S(k)+\boldsymbol{L} \Delta \boldsymbol{\Omega} \text { (19) }
$$

Because the value of $\boldsymbol{L} \Delta \boldsymbol{\Omega}$ is very small, so we have

$$
S(k+1) \approx e^{-|S(k)|} S(k)-\left(1-e^{-|S(k)|}\right) \operatorname{sign} S(k)
$$


So the controller (11) can satisfy the reaching condition (10).

In (19), the uncertain factor $\boldsymbol{L} \Delta \boldsymbol{\Omega}$ may let system state far away from the sliding surface. But as mentioned above, $e^{-|S(k)|}$ and $1-e^{-|S(k)|}$ of (19) possess the performance of adaptively adjusting reaching rate and restraining chattering phenomenon. So they can compensate the adverse effect produced by $\boldsymbol{L} \Delta \boldsymbol{\Omega}$. This completes the proof.

\section{SimUlATION STUDY}

In the section, we test the performance of the proposed algorithm by simulation. The simulation is based on a dumbbell network topology with a single bottleneck link [2]. We choose the Internet parameters as follows: $N=300, C=10 \mathrm{Mbps}$ (1 packet $=1000 \mathrm{bit}$, that is, $C=10^{4}$ packets $\left./ \mathrm{s}\right), R_{0}=50 \mathrm{~ms}$. The maximum buffer of the congestion router is 400 packets. The expected queue length $q_{0}$ is 200 packets. The initial queue length is 50 packets. According to the above parameters and (3), we can obtain

$$
\boldsymbol{A}=\left[\begin{array}{cc}
0 & 1 \\
-0.48 & -20.024
\end{array}\right], \boldsymbol{B}=\left[\begin{array}{c}
0 \\
-1.6667 \times 10^{11}
\end{array}\right],
$$

$p_{0}=7.2 \times 10^{-7}$. Let $T=10 \mathrm{~ms}, d=8, \tau_{1}=5 \mathrm{~ms}$, $l_{1}=1, \lambda=0.01$. By (4), we can obtain

$$
\begin{gathered}
\boldsymbol{G}=\left[\begin{array}{cc}
1 & 0.009 \\
-0.0043 & 0.8185
\end{array}\right], \boldsymbol{H}_{1}=\left[\begin{array}{l}
-2.0155 \times 10^{6} \\
-7.9297 \times 10^{8}
\end{array}\right], \\
\boldsymbol{H}_{2}=\left[\begin{array}{l}
-3.9649 \times 10^{6} \\
-7.5394 \times 10^{8}
\end{array}\right] .
\end{gathered}
$$

Under the same network condition, the controller in [10] is also simulated for the purpose of comparison.

\section{A. The Static Performance}

In this subsection, the Internet parameters are the same as the above settings, that is, these parameters are invariant. The simulation results are shown in Figure 3 and Figure 4.

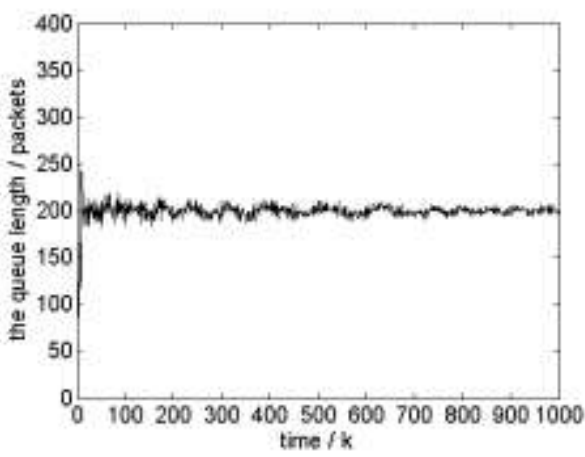

Figure 3. the queue length using the algorithm of [10].

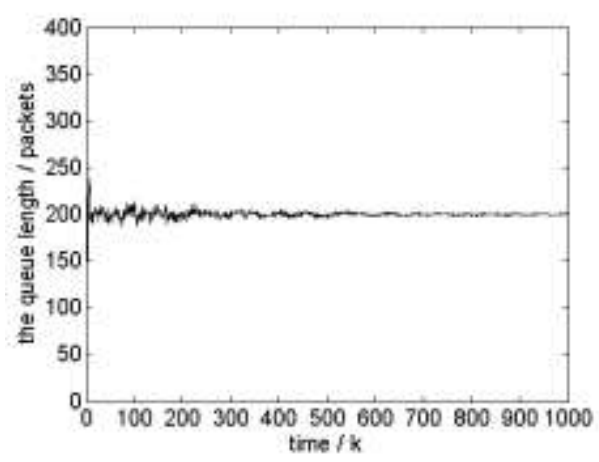

Figure 4. the queue length using the proposed algorithm.

As is shown in Figure 3 and Figure 4, the two algorithms all can make the queue length very close to the expected value. But the proposed algorithm possesses smaller steady state oscillation. This reason is that we design a better discrete reaching condition. The reaching condition can effectively restrain chattering phenomenon.

\section{B. The Robust Performance}

In this subsection, the simulation is done using the same parameters as the above settings, except that the change intervals of $N(t), C(t)$ and $R(t)$ are $40 \%$ of their nominal values, respectively. The simulation results are shown in Figure 5 and Figure 6.

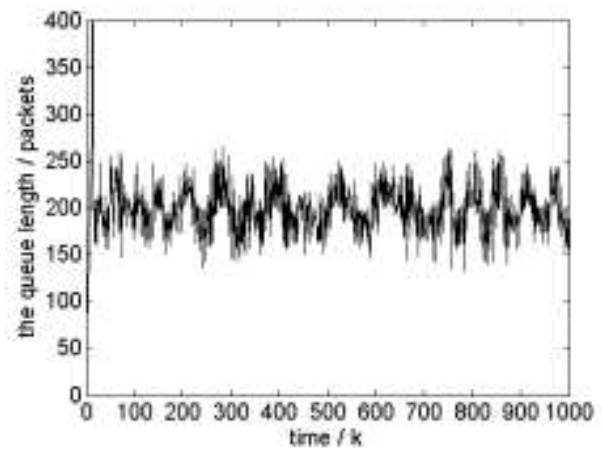

Figure 5. the queue length using the algorithm of [10].

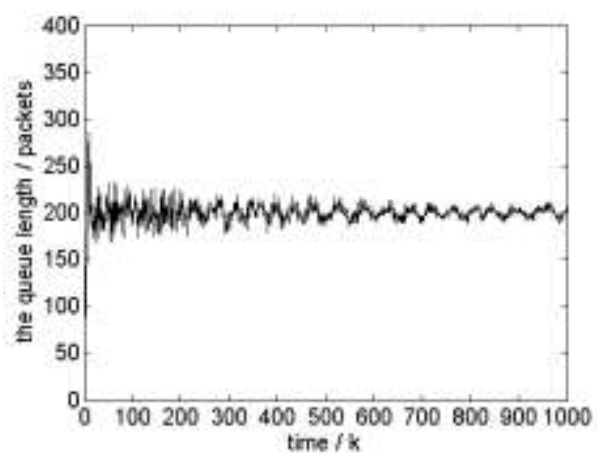

Figure 6. the queue length using the proposed algorithm.

As is shown in Figure 5 and Figure 6, the oscillation of the queue length becomes large when it uses the algorithm of [10]. This reason is that the effect of input time-delay factor was not considered in [10]. Because the system movement is a discrete global sliding mode, and 
the effect of uncertainties and time-delay factors are also considered, the proposed algorithm possesses better robustness. After a short regulating time, the queue length can quickly converge to about the expected value.

\section{CONCLUSION}

In order to solve the congestion problem in Internet, a discrete global sliding mode preestimate congestion control algorithm is proposed. The uncertain and timedelay factors are considered into the classical network model. So the precision of the model is improved. Then a discrete global sliding surface is designed. The robustness of the system is obviously improved. In addition, for reducing the chattering phenomenon in sliding mode system, a new discrete reaching condition is proposed. The controller that satisfied the reaching condition can effectively restrain the oscillation of the queue length in router. Simulation contrast show that the proposed algorithm possesses better stability and robustness.

\section{ACKNOWLEDGMENT}

This work was supported by Nature Science Foundation of Liaoning Province, China under Grant No. 201202092.

\section{REFERENCES}

[1] C. V. Hollot, V. Misra, D. Towsley, "A control theoretic analysis of RED”, Proceedings of IEEE INFOCOM, pp. 1510-1519, 2001.

[2] X. L. Chang, J. K. Muppala, "A stable queue-based adaptive controller for improving AQM performance", Computer Networks, vol. 50, no. 13, pp. 2204-2224, 2006.
[3] Z. Liu, Y. Zhang, C. L. Chen, "Adaptive mechanism-based congestion control for networked systems", International Journal of Systems Science, vol. 44, no. 3, pp. 533-544, 2013.

[4] H. Y. Sun, W. G. Jiang, "The stability studies of PSO-PID active queue management in wired network", Microelectronics \& Computer, vol. 31, no. 2, pp. 106-109, 2014.

[5] W. B. Gao, "Variable structure control of discrete-time systems", Acta Automatica Sinica, vol. 21, no. 2, pp. 154-161, 1995.

[6] F. J. Yin, C. J. Yue, "Study of TCP congestion control algorithm based on fuzzy tuning sliding mode surface", Computer Engineering and Applications, vol. 44, no. 15, pp. 85-87, 2008

[7] C. K. Chen, T. L. Liao, J. J. Yan, "Active queue management controller design for TCP communication networks: variable structure control approach", Chaos, Solitons \& Fractals, vol. 40, no. 1, pp. 277-285, 2009.

[8] W. C. Jiang, F. Y. Ren, R. Shu, "Sliding mode congestion control for data center Ethernet networks", IEEE International Conference on Computer Communications, pp. 1404-1412, 2012.

[9] S. G. Sajad, B. H. Mohamad, M. Mahdi, "Active queue management (AQM) for TCP/IP networks using discrete time sliding mode control (DSMC)", Proceedings of the 2005 IEEE Conference on Control Applications, pp. 727-730, 2005.

[10] M. Yan, T. D. K. Gugulovska, Y. W. Jing, G. Dimirovski, "Robust discrete-time sliding mode control algorithm for TCP networks congestion control", The 8th International Conference on Telecommunications in Modern Satellite, Cable and Broadcasting Services, pp. 393-396, 2007.

[11] C. Casetti, M. Meo, "A new approach to model the stationary behavior of TCP connections", IEEE International Conference on Computer Communications, pp. 367-375, 2000.

[12] Z. Wang, X. J. Guo, J. F. Si, Q. Zhang, "Discrete sliding mode control with time delay compensation for single input networked control systems", Systems Engineering and Electronics, vol. 28, no. 8, pp. 1237-1239, 2006.

[13] C. Y. Ye, Y. W. Jing, "Nonlinear active queue management based on adaptive global sliding mode control", Control and Decision, vol. 27, no. 10, pp. 1557-1560, 2012. 